\title{
A Survey on Dental Imaging for Building Classifier to Benefit the Dental Implant Practitioners
}

\author{
Shashikala $\mathbf{J}^{1}$ \\ Research Scholar, Department of Electronics and \\ Communication, JAIN (Deemed-to-be University), \\ Assistant Professor, BMS Institute of Technology \& \\ Management, Bengaluru, Karnataka, India
}

\author{
Thangadurai $\mathrm{N}^{2}$ \\ Professor and Research Coordinator, Department of \\ Electronics and Communication, Faculty of Engineering and \\ Technology, Centre for Emerging Technologies, JAIN \\ (Deemed-to-be University), Bengaluru, Karnataka, India
}

\begin{abstract}
Endo-osseous implants are considered an ideal dental fixture. It is becoming the preferred choice of the edentulous patient to rehabilitate toothlessness because of their aesthetic and functional outcome. Despite the successful surgery and implant placement, complications occur, which may be related to several factors, like operative assessment, treatment planning, patient-related factors, surgical procedures, and surgeons' experience. Comprehensive radiological assessment plays a vital role in clinical analysis for better treatment planning, avoiding complications, and increasing the Implant's success rate. However, despite the variety of dental imaging, choosing the right imaging technology has become difficult for clinical experts. The investigative survey conducted in this paper aims to determine the correlation between different imaging modalities, their essential role in implant therapy. This review extensively discussed which types of computational operations applied to image modalities in the existing literature address various noises and other relevant issues. These study findings reveal significant issues with various dental imaging modalities and provide an understanding to bridge all existing research gaps towards building cost-effective classification and predictive models for accurate dental treatment planning and higher implant success rates.
\end{abstract}

Keywords-Dental implant; complication; implant failure; dental imaging; pre-processing

\section{INTRODUCTION}

It is said that the mouth is a mirror of health that reflects the health condition of a person, or in other words, it is a cautionary system for disease. The mouth consists of both teeth and gums; their health condition is significant for oral health as poor oral health leads to various fatal diseases, too [1]. Apart from the fatal diseases, an issue of Edentulism(toothlessness) is found in both kids, adults, and old aged people due to respective reasons leads to the inconvenience of chewing the food so, poor nutritional intake and as a result a poor health condition [2]. However, the stage of Edentulism also creates an issue of the hollowness of speaking(pronunciation) along with other discomforts [3]. The traditional treatments of bridging and dentures were adopted for a long time as this was only a choice of treatment for the condition of Edentulism. However, the modern technique, namely dental implant surgery, is gaining popularity as an alternative solution to meet the deficiency of natural teeth by artificial tooth replacement [4].The dental implant procedure is based on the conception of direct contact between bone and the metal implant-(osseointegration), whose ultimate objective is to restore all the functional and aesthetic aspects [5]. An extensive planning and clinical examination performed by the dentist before surgery as a minor causality may cause serious harm to the patient. Therefore, an implant's success depends on several mutual factors, like implant region, bone quality, medical history of patient, skills, and the surgeon or dentist's experience. One of the significant challenges in dental implantation practice is the complex surgical procedures, which require preoperative and postoperative evaluation for achieving a higher success rate in dental implants [6]. The preoperative evaluation includes various factors such as the patient's general health conditions, bone quality, alveolar bone axis, and transplant site. The postoperative evaluation is carried out after the implantation to prevent any bias and risk of failure. Medical imaging technology plays a crucial role in the preoperative evaluation process. It provides the patient's anatomical details for the dental Implant-based on the maxillofacial structure and the two-dimensional geometric projection, helping clinical experts decide whether the implant surgery is suitable for the patient [7]. A systematic radiographic evaluation can provide an effective direction for precise positioning, which has important clinical significance in terms of accuracy and functional and aesthetic effects of the Implant [8]. Many imaging techniques are used in clinical dentistry practices, including conventional radiographic images and Computed Tomography (CT) for preoperative assessment and analysis of the complex jawbone structures. However, each imaging modality has some advantages and limitations too. Therefore, choosing the most suitable imaging method for dental implants is still tricky in dental practices. Another major issue is that the dental imaging is mostly associated with the poor image quality and superimposition factors that need to be process with an effective image enhancement and preprocessing techniques. In order to make the dental image representation more explanatory, several studies on dental image analysis have been conducted using digital image preprocessing methods. The proposed study aims to determine the prevalence of digital imaging modalities in dental implants and how they can help improvise the dental implant success rate. Therefore, this paper conducts a review analysis to highlight the importance of various imaging modalities and preprocessing techniques to explore the research gap. The rest of the sections of this paper are organized as follows: Section II discusses the background highlighting complications in the implant procedure and dental implant failures. This section also 
discusses how to improvise the dental implant success rate by prediction using image analysis. Section III presents a thorough analysis of what kinds of dental imaging are used and for what purposes. A comprehensive analysis is conducted on dental imaging modalities to highlight their importance and limitations in this section. Section IV presents an analysis of the current state-of-the-art, observing the trends towards adopting radiography and cone-beam computed tomography to avoid anatomical structures critical to dental implant surgery. Section $\mathrm{V}$ discussion and perspective are presented. In this, significant research direction based on evidential proofs, open research issues, and inferences is explored to develop effective predictive models to benefit dental implant practitioners. Finally, the overall contribution of this paper is concluded in Section VI.

\section{STUDY BACKGROUND}

Success and failure are two critical terms in dental implantology. The term implant success can be an ideal clinical setting, meaning that the Implant is into the jawbone and functions well and pleasingly. The term implant failure refers to the loss of osseointegration. Another statement is that it is an initial instance at which the Implant's efficacy, evaluated quantitatively, drops below a cut-off value or specified level [9].Dental implants may fail for various reasons, with the scope that distinguishes between complications and implant failures. This study uses the term implant failure, which is the complete loss of osseointegration, and the severity of implants that require to be removed from the implant site. In order to avoid any form of ambiguity, the study made a distinction between discussing implant failure and complications. Implant complications can be stated as an event that requires quantifiable clinical attention, and if such measures are not taken, the outcome of the implant therapy may be impaired. Implant complications may be caused due to poor patient selection, inadequate pre-assessment of the patient. Also, the degree of complications that are difficult to control may lead to implant failure. Therefore, through the proper patient selection and treatment planning, surgical fixing of implants can provide long-lasting functional and aesthetic restoration to the Edentulous Patient. Various studies have attempted to identify and quantify the rate of dental implant-related complications. However, to date, no single standard system for classifying dental implant-related complications. The authors in [10] discussed specific categories of complications related to dental implants. Existing studies [11-14] suggested the classification of complications associated with implant therapy considering all factors and causes. Other studies [15-16] considered the classification based on the particular phase of implant treatment that they tend to occur. The work carried out in [11] and [6] performed a classification of complications based on surgical, bone loss, implant loss, peri-implant soft tissue mechanical factor, and aesthetic/phonetic factor. In [13], the authors discussed the classification of dental implant complications, mechanical, technical, and biological. Classification of Surgical complications, Biological complications, and Restorative complications is carried out in [14]. The existing work of [15][16] discusses surgical complication based on three factors viz. i) implant treatment associated (wrong angulation, the judgment of improper implant-site, and lack of Communication among dental disciplines), ii) anatomy associated (nerve injury, bleeding, Sinus membrane complication, and devitalization of adjacent teeth), and iii) procedure associated-(Mechanical complication), lack of stability, mandibular fracture, aspiration, and ingestion. The authors in [17] discussed reversible complications are obstructions that are either temporary or easily fixed.

A proper surgical procedure analysis, including careful radiograph analysis, is significant to reduce the possibility of any implant complications and dental implant failure. Closer evaluation of dental radiographs helps to establish an appropriate treatment strategy for implant patients [18]. Several reviews and remarks have been given since the past few decades that described the significance of imaging techniques in dental disciplines [19-20]. Dental imaging plays a major-role in implant procedures to determine comprehensive information about the patient's maxillo-facial area to understand whether the surgical procedure is suitable for the patient. However, the role of imaging is not limited to determining only the maxillofacial area but also at different stages of the treatment processes, leading to the ease of surgical practice towards achieving higher success in dental implants [21-22].Imaging in dental treatment stage-1 subjected to patient diagnosis and clinical analysis conducted before implant surgery. Imaging evaluation assists the dentist in making a clinical decision and effective treatment planning based on past radiographs, medical history, and new radiographs evaluations that determine bone angulations, quality of bone, the critical structure of the maxillo-facials, presence of disease, and analysis of the implant site. In dental treatment phase-2, the role of imaging is to care about surgical intervention by assessing the surgical site and implant position during and after surgery and estimates the duration required for healing. Phase3 of dental treatment begins after the intra-operative assessment and continues until the Implant remains in the jaw. At this stage, dental imaging helps determine the care plan. If any changes or complications are noted during this period, the necessary clinical steps are taken to prevent any possibility of the risk of failure.

However, despite the variety of dental imaging, choosing an appropriate imaging technique has become a challenging task for clinical experts. Each imaging modality is associated with certain advantages and limitations. One of the major issues encountered in the dental image is the poor image quality due to poor contrast, uneven illumination, low resolution, and noise inclusion during the dental image acquisition process. In order to avoid any ill-effect, the radiation is kept low while taking the dental X-ray. The dental $\mathrm{X}$-ray constructed at low-radiation generates very poor-quality images with lower contrast and brightness, causing visibility differences during analysis. The specific noises during the radiography cause degradation to the dental image. [23]. Therefore, an effective mechanism should be implemented to enhance the quality of the image that can provide a significant clinical analysis in dental implant surgical procedures. The criteria that need to be considered as follows: 
- The dental image must provide cross-sectional interpretations that describe the spatial relationships between internal structures.

- The dental radiograph should not be compromised with distortion to a greater extent. However, the smallest distortion can be considered with a predictable average error to obtain a quantified analysis and precise measurement.

- It should provide an accurate description of bone density and cortical plate thickness to achieve the initial equilibrium and stability in the Implant.

- Radiography must provide higher dimensional accuracy in implant treatment procedure that includes analysis of implant placement site, pre-existing pathological condition of the patient, and evaluation alveolar thickness.

- The imaging tool should be available/provided at a reasonable price, and radiology doses should be as little as possible.

At present, there is various research works carried out towards dental imaging. However, there is always an impediment towards accurate diagnosis when it comes to medical image processing, as it demands a higher degree of accuracy. Hence, the prime statement of the problem of the proposed study is "To explore the strength and effectiveness of existing methodologies associated with dental imaging approach with respect to classification." The next section discusses about the different dental imaging modalities highlighting their advantage and limitations.

\section{Dental Imaging Modalities}

In this modern era, a variety of imaging technologies are widely used in the dental field. The traditional implant practitioners depend on $2 \mathrm{D}$ radiography. The advancement in imaging technology provided a 3D imaging technique, which offers advanced clinical evaluation in dental implants [24] and [25]. This section presents the adoption of verities of imaging modalities and their uses in different dental implant disciplines.

\section{A. Conventional Imaging Modalities}

Two-dimensional conventional imaging aims to complement the clinical analysis in dental implants by gaining a deep understanding of the internal teeth structure and alveolar bone. The different conventional imaging modalities are illustrated as follows:

- Periapical Radiography-It offers a systematic detail about the anatomical structures like teeth and surrounding tissues around the implant site. It is used for preoperative assessment to understand the implant area's structure, vertical height, and bone quality. However, these imaging modalities may be difficult to adopt due to accurate instrument positioning support's unavailability. This imaging technique is associated with distortion and magnification, limiting the quantified bone quality assessment, and suffers from providing accurate spatial relationships between internal overlapping dental structures [26].
- Cephalometric Radiography- This helps to capture the image of the head with the mandible in a lateral view to examine the associations between teeth, jaw, and the remaining part of the facial skeleton. This technique outlines the geometrical structure of the anterior alveolar region. The limitation is that it only displays cross-sectional images of bones associated with low magnification and overlapping issues [27].

- Panoramic Radiography- It is an x-ray radiography image that captures the entire mouth structure in a single image representation using a tomographic technique. It visualizes both maxillary-(upper jaw) and mandibular-(lower jaw) dental curves and supporting structures. It is mostly adopted as an initial screening $\mathrm{X}$-ray image to assess dental and bone support, identify affected teeth, and the condition of dental implants. This imaging technique is primarily used in the preoperative assessment to depict jaws in a single radiograph film or a charge-coupled device image receptor [28]. The distinct advantage of the panoramic imaging technique is that it offers a low patient radiation dose and is cost-effective in terms of time and computation complexity. It involves easy functioning and takes little time to capture the entire image of dentition in a single film or image receptor. Like other conventional radiography imaging techniques, it also has some limitations. Since this imaging technique is an extra oral technique, it does not provide delicate anatomy than periapical radiographs. It suffers from the issues like geometric distortion, superimposition, and magnification. Some other problems, like positioning error and technical/ processing error during panoramic radiography [29].

- Digital Radiography- It is direct digital radiography carried using several functional units that includes $\mathrm{x}$ ray-sensitive plates, sensors, mechanism of dividing it into electronic segments, and transferred to a computer to present and store the image. Compared to conventional imaging modalities, direct digital radiography offers good image quality with very little radiation. Few studies have mentioned that the overall reduction of radiation dose is up to $80 \%$ [30] and about $50 \%$ to $70 \%$ radiation reduction in intra oral and extra oral digital imaging [31]. Direct digital radiography has reduced processing time; images can be obtained immediately during the surgical procedure. Since this image is stored and processed in a computer, it can be manipulated with software programs to obtain enhanced visualization and accurate measurement. However, one of the significant disadvantages of digital radiography techniques is that the localization of sensors in the implant site sometimes becomes very challenging due to sensor size and positioning of the connecting cord.

Various conventional imaging modalities are discussed above. The limitation of conventional technology is that it encounters the superimposition of overlapping structures. The overlapping structure is caused due to the depiction of threedimensional maxillofacial structures onto a two-dimensional 
image plane, which results in the loss of spatial information that complicates the identification of objects of interest. The next sub-section discusses the applications and advantages of advanced imaging modalities in dental treatment.

\section{B. Advanced Imaging Modalities}

The conventional imaging modalities provide evidence for routine dentistry practices. Advanced imaging mechanisms are needed to demonstrate more information, complex diagnostics, and dental implant treatment plans. Hence, several techniques have changed the diagnosis and treatment planning strategies of dentistry. Some advanced dental imaging modalities are given below:

- Computerized Axial Tomography (CAT) - This is a unique X-ray imaging mechanism named computed tomography (CT), displaying the detailed images of the patient's anatomy with hard-and-soft tissues of the maxillofacial region. The CT uses multiple X-rays to construct a two-dimensional maxillofacial region and is converted into a three-dimensional image through processing. CT can obtain multiple, cross-sectional image-(slices) and generate high-contrast resolution images without suffering from superimposition and noise issues [32]. CT scans used to determine the quality of bones and the arrangement of teeth that cannot be efficiently obtained by the periapical imaging technique. CT identifies the diseases and immediacy of critical structures where implants are placed with the differentiation of tissues for analysis. The limitation of CT radiographs is that it has higher radiation exposure, high scan cost, and may not provide a good view of the small fissure resulting in false-negative readings [33].

- Magnetic Resonance Imaging (MRI) - MRI includes radio waves and adopts hydrogen atoms 'behaviour within a large magnetic field to look at body regions and generates an MR image of the internal structure. MRI represents soft tissue differences with high contrast sensitivity, which makes it advantageous over CT imaging. MR images can distinguish minor alveolar ducts and the contours between cortical bone and cancellous bone, thus obtaining necessary information about the maximum implant length, angle, and stability [34]. The MRI in the dental implant procedure seems to be an effective mechanism for 3-D imaging as it avoids the radiation risk of CT imaging. The adoption of MRI depends on the specific use conditions for an accurate diagnosis. The MRI achieves a flexible acquisition plane without changing image quality and resolution. However, MRI is susceptible to artifacts, distortion, and signal loss due to high magnetic susceptibility materials, while dental amalgam has little effect [35].

- Cone Beam Computed Tomography $(C B C T)-$ CBCT is a variation of conventional $\mathrm{CT}$. The application of CBCT is mainly for carrying diagnosis and planning of surgery in dental implants. One scan can produce many images of the area-of-interest. CBCT involves the mechanism of a cone-shaped-X-ray-beam moving around the patient to produce a large number of $2 \mathrm{D}$ views of ROI, and it is then converted into a $3 \mathrm{D}$ view using a cone-beam algorithm. CBCT in dentistry offers a high-resolution representation of bone and teeth, giving a spatial relationship between the adjacent structures. CBCT is used to evaluate osseous disease and identify jaw bone infections and diseases that help perform risk-free surgery, i.e. complications (pain and swelling) [36]. CBCT includes fast scanning procedures associated with lower radiation dose, lower scan cost and DICOM compatibility and has reduced metal product interference than other methods [37-38]. The limitation of CBCT is that it has a limited contrast range, gives fewer details of internal soft tissues, and has a large noise factor and artifacts.

\section{Primary Findings}

All the imaging methods have a vital role in dentistry applications. The conventional 2D and advanced 3D radiographs provide necessary information for dental treatment and Implant, while a dental digital panoramic image can offer a clinical diagnosis of the jawbone. The significance of digital panoramic imaging is that it has a low radiation dose and shorter exposure time [39]. But intraoral imaging has issues like low image quality, variable magnification, and ghost images. The superposition of the upper cervical spine is the main limitation of panoramic X-ray photography [40], and osseointegration cannot be detected due to overlapping issues [41]. Hence, it is limited to preoperative diagnostic, leading to implant failure [42]. Hence, implantation surgery may compromise the health of nearby soft tissues and cells [43]. The use of CT and CBCT is described in [44-45] over 2D radiographs to assess complex structures like the maxillary sinus. However, the limitations of these imaging modalities are i) not available in many local hospitals due to higher cost and multi-disciplinary technical requirements. The researchers also informed that the patients were exposed to higher radiation doses when CT examination is done than of 2D digital imaging and CBCT examination. Some research works also compared CBCT and digital panoramic imaging to assess the bone height towards planning treatment in different dental implant phases [46] and revealed that digital panoramic is self-sufficient to describe the incisor area but lacks in the canine area. Also, [47] have performed a comparison of error estimation and found CBCT has a better result, which holds a low average preoperative assessment error in the maxillary area than the digital panoramic imaging technique.

\section{STATE-OF-THE-ART REVIEWS}

This section presents a review study on the state-of-the-art in the context of digital radiographs adopted in dental implant surgery. Digital radiography is cost-effective and is used in dental radiography. The study (Choi et al. [48]) investigates the impact of enhancement over periapical radiographs by considering three pre-processing techniques for diagnostics. The outcome gives quality differences between the processed image and the input image. A work of (Hao et al. [49]) considered denoising CBCT dental images where improved non-local means filtering is applied [49]. The outcomes demonstrated in terms of PSNR and MSE. The segmentation 
operation over digital radiograph image is performed in (Cunha et al.) for the accurate visualization of dental Implant and crestal bone line [50]. A contrast enhancement over Digitized film-based panoramic dental image using the CLAHERayleigh is found in the study of (Suprijanto et al.). The study outcome shows that this method has achieved better performance in terms of PSNR [51]. The authors in the study of (Yin et al.) have used approaches of noise filtering technique for CBCT image based on thresholding mechanisms and wavelet transform [52]. (Mortaheb and Rezaeian) introduces an automated dental CT image approach for identifying the vertical structure and arrangement of the teeth [53]. The study (Lamecker et al.) focuses on automated segmentation operation for Computer-assisted craniomaxillo facial surgery using conebeam volumetric tomography-(CBVT) dental image [54]. A noise that occurred by positioning error in the digital panoramic dental image is considered in the work of (Amiri and Moudi et al.) and (Kandan and Kumar), which achieves better visualization of the roots of maxillary teeth in the digital radiograph [55-56]. The work carried out by (Naik et al.) used the histogram equalization technique for enhancing the overall visualization of the digital radiographs for accurate analysis of the bone structure and quality [57]. The authors (Kamezawa et al.) used a multiple noise filtering approach for CBCT imaging for exposure radiation dose reduction in an automated guided patient positioning system [58]. An edge enhancement-based pre-processing technique is applied on panoramic X-Ray in the study (Jufriadif et al.) to detect proximal caries [59]. The work of (Supriyanti et al.) used a point processing mechanism for contract stretching of a digital panoramic dental image [60]. In the study of (Khatter et al.), the authors have applied a multiscale retinex mechanism over CBCT to perform a precise assessment of root canal anatomy for endodontic therapy [61]. An image pre-processing I2I scheme based on neural network architecture is adopted in the research work of (Zhao et al.), which considers generative adversarial networks (GAN) to suppress ring artifacts [62]. Mean-shift algorithm-based image segmentation is adopted in the study of (Gunawan et al.). The authors have identified a fuzzy region in the segmented image and performed fuzzy merging processes based on similarity measurement [63]. A work towards brightness preserving in dental digital periapical images using entropy and histogram analysis is found in the study of (Qassim et al.) [64]. A most recent research work carried out by (Abdallah et al.) [65] have used Anisotropic filtering to eliminate noise, and Contrast Limiting Adaptive Histogram Equalization (CLAHE) to enhance contrast, and sharpness of the dental panoramic image.

\section{Discussion AND PERSPECTIVE}

Several radiographic modalities were described with their respective features and limitations. Each has its applicability in respective dental conditions to assist the dentists in planning, evaluation, and implant treatment. A precise strategy can reduce the surgical complexity and postoperative complications and lead to higher success considering both aesthetic and functional aspects. Therefore, suitable radiographic selection plays an important role, and the advanced 3D radiograph technique provides all the functional utilities compared to the conventional radiograph technique. Due to the cost factor, digital, panoramic radiography is in wide use. However, advanced imaging modalities like (MRI, CT, and CBCT) provide better visualization and compatibility with analysis tools so that many complementary and significant information for successful dental implant planning is made available. The MRI facilitates precise localization of the complex structures and useful when the differentiation of soft tissue analysis is requiring, but it carries artifacts like geometric distortion. CT imaging is more suitable for the analysis of bone quantity and quality because it can quickly cover the expanded anatomical area and generate images with reduced noise caused by the patient's movement. The advanced and recent modality, namely, Cone Beam CT (CBCT), offers fast data acquisition of the complete field of view with minimal radiation exposure. It is useful in the diagnosis and Endodontic treatment. In all the above discussed, dental imaging modalities suffer image quality degradation due to various factors like superimposition, geometric distortion, loss of signal, contrast, motion artifacts, and positioning errors that cause challenges during interpretation. The efficient pre-processing techniques can enhance image quality; thereby, significant interpretations for accurate treatment planning in the pre-assessment phase during surgery can be achieved. The post-surgery complication can be avoided to illuminate the possibilities of implant failure.

A systematic review of existing research literature with these imaging modalities is inferred, used while proposing models for segmentation of ROI and classification of complex anatomical structures of the oral region. This paper potentially identifies the trend of the pre-processing techniques adopted and also found that both 2-D dental radiographs and CBCT are advantageous over other modalities. It is recommended that adopting 2D dental imaging with an efficient pre-processing technique for enhancement will be a better choice in implant treatment planning and surgical process until CBCT matures. In the future, CBCT with efficient pre-processing for enhancement and noise filtering may provide a way better path towards an effective modality for successful dental implantation.

\section{A. Research Gap}

Based on the above discussion and review analysis, the significant open research problem is highlighted as follows:

No standard open-source dataset is available for the analysis of CBCT. In most research works, the dataset was either collected from the hospitals or considered based on the experimental setup. It has also been seen that few research works have considered dental image data from internet sources.

- Most image enhancement techniques are in the transform domain so that some artifacts may appear in the output image. As a result, it may lead to overenhancement and issues related to the edge of the image.

- Lack of novelty is analyzed in most of the existing literature subjected to dental image pre-processing tasks. Most of the existing research works follow a similar pattern towards pre-processing the medical image. An improvement and optimization mechanism should be considered. 
- The research works towards a predictive model have also not focused on the computational complexity associated with their prediction model for classification of the anatomical structure in preoperative assessment for the Dental Implant.

- Analysis of dental Images based on consideration of suitable parameters is missing in the existing literature. In order to perform effective image analysis, researchers must Analysis and evaluate image quality based on the HSV feature and statistics error metrics like Peak-Signal-to-noise-ratio, MSE-(Mean square error), SNR-(Signal to noise ratio), CNR-(Contrast to noise ratio), SD-(Spectral Distance) and SSIM(Structural Similarity index.).

- Standard benchmarking is also missing in most of the existing image pre-processing methods.

\section{CONCLUSION}

A dental implant is a complicated procedure that involves multi-disciplinary activities for treatment and surgical planning. Appropriate knowledge and understanding of the complexity and evaluation of implant failure factors is crucial for dental practitioners. Apart from this, digital imaging analysis is critical stage clinicians need to understand the technical parameters. However, equally, it is essential to manipulate these dental radiographs using a suitable preprocessing mechanism to know the potential factors associated with each stage of implant treatment. This paper has presented an investigative review analysis of different complications factors, various dental imaging modalities, and state-of-art preprocessing techniques. Finally, the proposed survey also explored the significant issues in the existing literature and discussed the significant point of highlighting the open research problem. Therefore, the proposed review works provide an effective future research direction for establishing predictive models with effective pre-processing schemes to benefit dental implant practitioners.

\section{REFERENCES}

[1] Clark, Danielle, and Liran Levin. "In the dental implant era, why do we still bother saving teeth?." Dental Traumatology 35, no. 6 (2019): 368375 .

[2] Andersson, Lars, Jens O. Andreasen, Peter Day, Geoffrey Heithersay, Martin Trope, Anthony J. DiAngelis, David J. Kenny et al. "Guidelines for the Management of Traumatic Dental Injuries: 2. Avulsion of Permanent Teeth." Pediatric dentistry 37, no. 6 (2015).

[3] Emami, Elham \& de Souza, Raphael \&Kabawat, Marla \&Feine, Jocelyne. (2013). The Impact of Edentulism on Oral and General Health. International journal of dentistry. 2013. 498305. 10.1155/2013/498305.

[4] Alajlan, Abdulrahman, AryafAlhoumaidan, AbeerEttesh, and Mazen Doumani. "Assessing Knowledge and Attitude of Dental Patients regarding the Use of Dental Implants: A Survey-Based Research." International journal of dentistry 2019 (2019).

[5] Oh, Ji-hyeon. "Recent advances in dental implants." Maxillofacial plastic and reconstructive surgery 39, no. 1 (2017): 33.

[6] Bryce, G., D. I. Bomfim, and G. S. Bassi. "Pre-and postoperative management of dental implant placement. Part 2: management of earlypresenting complications." British dental journal 217, no. 4 (2014): 171.

[7] Gupta, Sarika, Neelkant Patil, Jitender Solanki, Ravinder Singh, and Sanjeev Laller. "Oral implant imaging: a review." The Malaysian journal of medical sciences: MJMS 22, no. 3 (2015): 7.
[8] Jayadevappa, Busnur Shilpa, G. S. Kodhandarama, and S. V. Santosh. "Imaging of dental implants." Journal of Oral Health Research 1, no. 2 (2010): 50-62.

[9] Esposito M, Hirsch JM, Lekholm U, Thomsen P. Biological factors contributing to failures of osseointegrated oral implants (I) success Criteria and Epidemiology. Eur J Oral Sci 1998;106:527-51;

[10] Hanif, Ayesha, Saima Qureshi, Zeeshan Sheikh, and Haroon Rashid. "Complications in implant dentistry." European journal of dentistry 11, no. 01 (2017): 135-140.

[11] Goodacre CJ, Kan JY, Rungcharassaeng K. Clinical complications of osseointegrated implants. J Prosthet Dent 1999;81(5):537-552.

[12] Goodacre CJ, Bernal G, Rungcharassaeng K, Kan JYK. Clinical complications with implants and implant prostheses. J Prosthet Dent 2003;90(2):121-132.

[13] Hanif, Ayesha, Saima Qureshi, Zeeshan Sheikh, and Haroon Rashid. "Complications in implant dentistry." European journal of dentistry 11, no. 01 (2017): 135-140.

[14] Guo, Q., R. Lalji, A. V. Le, R. B. Judge, D. Bailey, W. Thomson, and K. Escobar. "Survival rates and complication types for single implants provided at the Melbourne Dental School." Australian dental journal 60, no. 3 (2015): 353-361.

[15] Misch K, Wang H. Implant surgery complications: etiology and treatment. Implant Dent 2008;17(2):159-168.

[16] Misch, Kelly, and Hom-Lay Wang. "Implant surgery complications: etiology and treatment." Implant dentistry 17, no. 2 (2008): 159-168.

[17] (60) Park SH, Wang HL. Implant reversible complications: classification and treatment. Impl Dent 2005;14:211-220.

[18] Gupta, Sarika, Neelkant Patil, Jitender Solanki, Ravinder Singh, and Sanjeev Laller. "Oral implant imaging: a review." The Malaysian journal of medical sciences: MJMS 22, no. 3 (2015): 7.

[19] Vandenberghe, Bart. "The digital patient-Imaging science in dentistry." Journal of dentistry 74 (2018): S21-S26.

[20] Satpathy, Anurag, Rajeev Ranjan, SubhashreePriyadarsini, Somesh Gupta, Piyush Mathur, and Monalisa Mishra. "Diagnostic Imaging Techniques in Oral Diseases." In Medical Imaging Methods, pp. 59-95. Springer, Singapore, 2019.

[21] Vandenberghe, Bart. "The digital patient-Imaging science in dentistry." Journal of dentistry 74 (2018): S21-S26.

[22] Chandak, Shruti, Arjit Agarwal, Ashutosh Kumar, Rajul Rastogi, Pawan Joon, Asif M. Wani, and Yuktika Gupta. "Comparative Study of DENTA Scan and Radiography for Preoperative Assessment of Dental Implants." Annals of International Medical and Dental Research 4, no. 1 (2018): 26.

[23] Rahmi-Fajrin H, Puspita S, Riyadi S, Sofiani E. Dental radiography image enhancement for treatment evaluation through digital image processing. Journal of clinical and experimental dentistry. 2018 Jul;10(7):e629.

[24] White, Stuart C., and Michael J. Pharoah. "The evolution and application of dental maxillofacial imaging modalities." Dental Clinics of North America 52, no. 4 (2008): 689-705.

[25] Ríos-Santos, José V., Cristina Ridao-Sacie, Pedro Bullón, Ana Fernández-Palacín, and Juan J. Segura-Egea. "Assessment of periapical status: a comparative study using film-based periapical radiographs and digital panoramic images." Med Oral Patol Oral Cir Bucal 15, no. 6 (2010): e952-6.

[26] Tanwani, HemlataBhagwan, Sheetal Sameer Potnis, Sandesh Satish Baralay, and Sameer Sidagouda Patil. "Comparison of conventional and digital cephalometric analysis: A pilot study." Journal of Dental and Allied Sciences 3, no. 2 (2014): 80.

[27] Choi, Bo-Ram, Da-Hye Choi, Kyung-Hoe Huh, Won-Jin Yi, Min-Suk Heo, Soon-Chul Choi, Kwang-Hak Bae, and Sam-Sun Lee. "Clinical image quality evaluation for panoramic radiography in Korean dental clinics." Imaging science in dentistry 42, no. 3 (2012): 183-190.

[28] Jayachandran, Sadaksharam. "Digital imaging in dentistry: A review." Contemporary clinical dentistry 8, no. 2 (2017): 193.

[29] Langland OE, Langlais RP, Preece JW. Principles of dental imaging. 2nd ed. Philadelphia: Lippincott Williams \& Wilkins, 2002: 285. 
[30] Farman AG, Farman TT. Extraoral and panoramic systems. Dent Clin North Am 2000; 44: 257-272,

[31] Aggarwal V, Logani A, Shah N. The evaluation of computed tomography scans and ultrasounds in the differential diagnosis of periapical lesions.

[32] Shah, Naseem, Nikhil Bansal, and Ajay Logani. "Recent advances in imaging technologies in dentistry." World journal of radiology 6, no. 10 (2014): 794.

[33] Niraj, Lav Kumar, Basavaraj Patthi, Ashish Singla, Ritu Gupta, Irfan Ali, Kuldeep Dhama, Jishnu Krishna Kumar, and Monika Prasad. "MRI in dentistry-a future towards radiation free imaging-systematic review." Journal of clinical and diagnostic research: JCDR 10, no. 10 (2016): ZE14.

[34] Mendes, Silwan, Carin A. Rinne, Julia C. Schmidt, Dorothea DagassanBerndt, and Clemens Walter. "Evaluation of magnetic resonance imaging for diagnostic purposes in operative dentistry - a systematic review." Clinical Oral Investigations (2019): 1-11.

[35] Bornstein, Michael M., William C. Scarfe, Vida M. Vaughn, and Reinhilde Jacobs. "Cone beam computed tomography in implant dentistry: a systematic review focusing on guidelines, indications, and radiation dose risks." International journal of oral \& maxillofacial implants 29 (2014).

[36] Gupta, Jyoti, and Syed Parveez Ali. "Cone beam computed tomography in oral implants." National journal of maxillofacial surgery 4, no. 1 (2013): 2 .

[37] Bornstein, Michael M., Keith Horner, and Reinhilde Jacobs. "Use of cone beam computed tomography in implant dentistry: current concepts, indications and limitations for clinical practice and research." Periodontology 2000 73, no. 1 (2017): 51-72.

[38] Jacobs, Reinhilde, Benjamin Salmon, Marina Codari, Bassam Hassan, and Michael M. Bornstein. "Cone beam computed tomography in implant dentistry: recommendations for clinical use." BMC Oral Health 18, no. 1 (2018): 88.

[39] Suomalainen A, PakbaznejadEsmaeili E, Robinson S. Dentomaxillofacial imaging with panoramic views and cone beam CT. Insights Imaging 2015; 6: 1-16.

[40] Tang Z, Liu X, Chen K. Comparison of digital panoramicradiography versus cone beam computerized tomography for measuring alveolar bone. Head Face Med 2017; 13: 2.

[41] Isidor F. Clinical probing and radiographic assessment in relation to the histologic bone level at oral implants in monkeys. Clin Oral Implants Res 1997; 8: 255-64.

[42] Greenstein G, Cavallaro J, Romanos G, Tarnow D. Clinical recommendations for avoiding and managing surgical complications associated with implant dentistry: a review. J Periodontol 2008; 79: 1317-29.

[43] Tang Z, Liu X, Chen K. Comparison of digital panoramic radiography versus cone beam computerized tomography for measuring alveolar bone. Head Face Med 2017; 13: 2.

[44] Hassan B, Jacobs R. Cone beam computed tomography - 3D imaging in oral and maxillofacial surgery. Eur Med Imaging Rev 2008; 1: 38-40.

[45] Kopecka D, Simunek A, Streblov J, Slezak R, Capek L. Measurement of the interantral bone in implant dentistry using panoramic radiography and cone beam computed tomography: a human radiographic study. West Indian Med J 2014; 63: 503-9.

[46] Renton T, Dawood A, Shah A, Searson L, Yilmaz Z. Post-implant neuropathy of the trigeminal nerve. A case series. Br Dent J 2012; 212 : E17.

[47] Angelopoulos C, Thomas S, Hechler S, Parissis N, Hlavacek M. Comparison between digital panoramic radiography and conebeam computed tomography for the identification of the mandibular canal as part of presurgical dental implant assessment. J Oral Maxillofac Surg 2008; 66: 2130-5.

[48] Choi, Jin-Woo, Won-Jeong Han, and Eun-Kyung Kim. "Image enhancement of digital periapical radiographs according to diagnostic tasks." Imaging science in dentistry 44, no. 1 (2014): 31-35.

[49] Hao, Jia, Li Zhang, Liang Li, and Kejun Kang. "An improved non-local means regularized iterative reconstruction method for low-dose dental
CBCT." In 2012 IEEE Nuclear Science Symposium and Medical Imaging Conference Record (NSS/MIC), pp. 3422-3425. IEEE, 2012.

[50] Cunha, Pedro, Miguel A. Guevara, Ana Messias, Salomão Rocha, Rita Reis, and Pedro MG Nicolau. "A method for segmentation of dental implants and crestal bone." International journal of computer assisted radiology and surgery 8, no. 5 (2013): 711-721.

[51] Juliastuti, E., and LusiEpsilawati. "Image contrast enhancement for filmbased dental panoramic radiography." In 2012 International Conference on System Engineering and Technology (ICSET), pp. 1-5. IEEE, 2012.

[52] Yin, Yong, Gang Yu, Hongjun Wang, Zhi Liu, and Dengwang Li. "CBCT image denoising based on multi-scale wavelet transform." In 2010 3rd International Conference on Biomedical Engineering and Informatics, vol. 1, pp. 150-153. IEEE, 2010.

[53] Mortaheb, Parinaz, Mehdi Rezaeian, and Hamid Soltanian-Zadeh. "Automatic dental CT image segmentation using mean shift algorithm." In 2013 8th Iranian Conference on Machine Vision and Image Processing (MVIP), pp. 121-126. IEEE, 2013.

[54] Lamecker, Hans, Stefan Zachow, Antonia Wittmers, Britta Weber, H. Hege, B. Isholtz, and Michael Stiller. "Automatic segmentation of mandibles in low-dose CT-data." International Journal of Computer Assisted Radiology and Surgery 1 (2006): 393.

[55] AsadiAmiri, Sekine, and Ehsan Moudi. "Image quality enhancement in digital panoramic radiograph." Journal of AI and Data Mining 2, no. 1 (2014): 1-6.

[56] Kandan, R. Somas, A. John, and S. Kumar. "An improved contrast enhancement approach for panoramic dental x-ray images." ARPN J Eng App Sci 10 (2015): 1897-1901.

[57] Naik, Anjali, Shubhangi Vinayak Tikhe, and S. D. Bhide. "Histogram Equalization for Class-Identification of Dental Disease Using Digital Radiography." In International Conference on Business Administration and Information Processing, pp. 144-151. Springer, Berlin, Heidelberg, 2010.

[58] Kamezawa, Hidemi, KatsutoshiShirieda, Hidetaka Arimura, Noboru Kameda, and Masafumi Ohki. "An approach of exposure dose reduction of cone-beam computed tomography in an image guided patient positioning system by using various noise suppression filters." In 2014 Joint 7th International Conference on Soft Computing and Intelligent Systems (SCIS) and 15th International Symposium on Advanced Intelligent Systems (ISIS), pp. 1475-1780. IEEE, 2014.

[59] Na'am J, Harlan J, Madenda S, Santony J, Suharinto C. Detection of proximal caries at the molar teeth using edge enhancement algorithm. International Journal of Electrical and Computer Engineering. 2018 Oct $1 ; 8(5): 3259$.

[60] Supriyanti R, Setiadi AS, Ramadhani Y, Widodo HB. Point Processing Method for Improving Dental Radiology Image Quality. International Journal of Electrical and Computer Engineering (2088-8708). 2016 Aug $1 ; 6(4)$.

[61] Khatter, Ashish, Anita Thakur, and Nitya Reddy. "CBCT Image Feature Enhancement for Endodontic Therapy." In 2019 6th International Conference on Signal Processing and Integrated Networks (SPIN), pp. 293-296. IEEE, 2019.

[62] Zhao, Shuyang, Jianwu Li, and QirunHuo. "Removing ring artifacts in CBCT images via generative adversarial network." In 2018 IEEE International Conference on Acoustics, Speech and Signal Processing (ICASSP), pp. 1055-1059. IEEE, 2018.

[63] Gunawan W, Arifin AZ, Indraswari R, Navastara DA. Fuzzy Region Merging Using Fuzzy Similarity Measurement on Image Segmentation. International Journal of Electrical \& Computer Engineering (20888708). 2017 Dec 1;7(6).

[64] Qassim, Hassan M., Nasseer M. Basheer, and Mazin N. Farhan. "Brightness preserving enhancement for dental digital X-ray images based on entropy and histogram analysis." J Appl Sci Eng 22 (2019): 187-94.

[65] Yousif Mohamed, Nouf $\mathrm{H}$ Abuhadi, and Maryam Hasan Hugri"Enhancement of Dental X-rays Images Using Image Processing Techniques"Journal of Research in Medical and Dental Science 2021, Volume 9, Issue 2, Page No: 12-16. 